\title{
Editorial: analysis in software engineering
}

\author{
Robert J. Hall
}

Received: 10 February 2012 / Accepted: 15 February 2012 / Published online: 22 February 2012

(C) Springer Science+Business Media, LLC 2012

Analysis, whether it be program analysis, requirements analysis, or domain analysis, is, of course, a core concern in software engineering. Since analysis problems are based on fundamentally uncomputable computational problems, the best we can hope for is to find practical approximate or heuristic tools that can add significant value to our human analytic reasoning. And since the problems are so difficult, it will always be possible, though not necessarily easy, to improve our tools' performance.

This regular issue showcases three contributions relating to analytic concerns in software engineering. The first two deal with verification and validation of programs using two very different approaches. The third aids human analysts in extracting useful formal model information out of unstructured sources.

- Symbolic execution of a program can help in verification and validation by characterizing the behavior of a program on a whole (infinite) class of cases by generalizing over a single input. Lazy symbolic execution generalizes more traditional approaches in order to handle complexities like aliasing resulting from use of dynamic heap objects. In "Efficient and formal generalized symbolic execution", Deng, Lee, and Robby describe improved algorithms for lazy symbolic execution and report on extensive comparisons of the improved algorithms to the traditional one, showing significant improvement.

- Testing is another approach to verifying that a program performs correctly on particular inputs. However, to automate testing it is necessary to have an oracle that can judge computed outputs for correctness. In "Artificial neural networks as multinetworks automated test oracles", Shahamiri, Wan-Kadir, Ibrahim, and Hashim show an improved way to employ neural networks to learn test oracles. The key

R.J. Hall (殴

AT\&T Labs Research, Florham Park, NJ 07932, USA

e-mail: Bob.ASEJ@gmail.com 
idea is to use separate neural networks to learn separate output values, rather than to attempt to train a single neural net to produce all outputs correctly.

- Software product lines allow representing and generating a family of software products that are related by being different combinations and instances of various domain features. To manage the complexity of building SPL artifacts, a common approach is to build a feature model that describes variation points, constraints, and requirements for combining features to form family members of the SPL. In "Decision support for the software product line domain engineering lifecycle", Bagheri, Ensan, and Gasevic integrate a number of data mining and natural language techniques to assist in building and maintaining product line feature models, helping in extracting domain knowledge from natural language documents and other sources, and recording traceability links. Their case study shows that this tool suite does indeed significantly help domain analysts in the many tasks necessary to carry out this complex activity.

Please enjoy these contributions and feel free to email the authors with any questions. You are invited, as always, to email me at Bob.ASEJ@gmail.com with your thoughts on this issue or the Journal in general. 